\title{
Promotive role of microRNA-150 in hippocampal neurons apoptosis in vascular dementia model rats
}

\author{
CHENGQUN WEI $^{1 *}$, XIUZHI XU ${ }^{1 *}$, HONGYAN ZHU $^{1}$, XIUYAN ZHANG $^{2}$ and ZHAN GAO ${ }^{1}$ \\ Departments of ${ }^{1}$ General Practice and ${ }^{2}$ Neurology, Heilongjiang Provincial Hospital, Harbin, Heilongjiang 150001, P.R. China
}

Received June 17, 2020; Accepted December 21, 2020

DOI: $10.3892 / \mathrm{mmr} .2021 .11896$

\begin{abstract}
Cognitive impairment is one of the primary features of vascular dementia (VD). However, the specific mechanism underlying the regulation of cognition function in VD is not completely understood. The present study aimed to explore the effects of microRNA (miR)-150 on VD. To determine the effects of miR-150 on cognitive function and hippocampal neurons in VD model rats, rats were subjected to intracerebroventricular injections of miR-150 antagomiR. The Morris water maze test results demonstrated that spatial learning ability was impaired in VD model rats compared with control rats. Moreover, compared with antagomiR negative control (NC), miR-150 antagomiR alleviated cognitive impairment and enhanced memory ability in VD model rats. The triphenyltetrazolium chloride, Nissl staining and immunohistochemistry results further demonstrated that miR-150 knockdown improved the activity of hippocampal neurons in VD model rats compared with the antagomiR NC group. To validate the role of miR-150 in neurons in vitro, the $\mathrm{PC} 12$ cell line was used. The flow cytometry and Hoechst 33342/PI double staining results indicated that miR-150 overexpression significantly increased cell apoptosis compared with the mimic NC group. Moreover, the dual-luciferase reporter gene assay results indicated that miR-150 targeted HOXA1 and negatively regulated HOXA1 expression. Therefore, the present study indicated that miR-150 knockdown ameliorated VD symptoms by upregulating HOXA1 expression in vivo and in vitro.
\end{abstract}

Correspondence to: Mrs. Hongyan Zhu, Department of General Practice, Heilongjiang Provincial Hospital, 82 Zhongshan Road, Xiangfang, Harbin, Heilongjiang 150001, P.R. China

E-mail: hongyanzhu3192@163.com

Mrs. Xiuyan Zhang, Department of Neurology, Heilongjiang Provincial Hospital, 82 Zhongshan Road, Xiangfang, Harbin, Heilongjiang 150001, P.R. China

E-mail: xyzhang0973@163.com

*Contributed equally

Key words: vascular dementia, microRNA-150, homobox A1, hippocampal neurons, cognitive impairment, microRNA-150 antagomiR

\section{Introduction}

Vascular dementia(VD) is one of the leading causes of dementia following Alzheimer's disease (AD), which accounted for 15\% of all dementia cases following AD in 2015 worldwide (1). At present, there is a shortage of accepted treatments and diagnostic approaches for the management of VD (2). VD is characterized by neurocognition dysfunction and behavioral alterations indicative of cognitive impairment (3). Basic and clinical studies have demonstrated that progressive neuronal loss is closely related to dementia (4). Atherosclerosis, infarction and arteriosclerosis are markers of neurodegeneration in VD (5). Although different drugs, including donepezil and galantamine, are effective in curing AD, the effects of the drugs significantly vary among individuals when used as VD therapies (6). The impact of molecular mechanisms on brain studies has been increasingly investigated, and several researchers developing novel therapeutic strategies for dementia have focused on microRNA (miRNA/miR)-based mechanisms aiming to identify further targets for the diagnosis of VD (7-9).

miRNAs are single-stranded RNAs, 22 nucleotides in length, that can mediate mRNA expression (10). miRNAs are associated with the regulation of type 2 diabetes mellitus, ischemic stroke, AD and VD (8). For instance, downregulation of angiogenic miR-126 leads to cognitive impairment in VD model mice (11). miR-150 has been reported to serve a role in inflammation and different neurological syndromes in association with the potential downstream targets (12). Moreover, miR-150 is one of the miRNAs correlated with mice displaying cognitive deficits and advanced neuropathology (13), indicating a possible correlation with neuronal dysfunction. In addition, long non-coding RNA myocardial infarction associated transcript knockdown is responsible for progressive neuronal loss and neurodegeneration, as well as behavioral deficits in $\mathrm{AD}$, which is associated with miR-150 overexpression (14). However, how miR-150 functions in VD, as well as its role in mediating neuronal apoptosis in vitro and cognitive function in vivo are not completely understood. Homeobox (HOX)A1, a member of the HOX family, is involved in the development of vertebrates, as well as in multiple diseases, such as atherosclerosis (15). The targeting relationship between miR-339-5p and HOXA1 has been observed in ischemic brain damage (16). However, to the best of our knowledge, an association between miR-150 and 
HOXA1 has not been previously reported. Furthermore, the role of HOXA1 in VD is not completely understood. Therefore, the present study hypothesized that miR-150 may serve as a regulator of VD by interacting with HOXA1. To investigate the hypothesis, miR-150 knockdown was established using miR-150 antagomiR, with an attempt to utilize miRNAs as biomarkers and develop a novel miRNA-based therapy for patients with VD.

\section{Materials and methods}

Ethics statement. The present study was approved by the Laboratory Animal Care and Use Committee of Heilongjiang Provincial Hospital (approval no. KY-2018-197) and performed according to the Guidelines for the Care and Use of Laboratory Animals published by the National Institutes of Health (17).

Establishment of VD model rats. A total of 48 male Sprague-Dawley rats (weight, 180-220 g; age, 6-7 weeks) were provided by the Laboratory Animal Center of Heilongjiang Provincial Hospital. Rats were housed at $25 \pm 1^{\circ} \mathrm{C}$ with $50-70 \%$ humidity, 12-h light/dark cycles, and free access to food and water. They were acclimated to the housing conditions for 7 days before modeling.

To establish VD model rats, rats were subjected to global cerebral ischemia via permanent occlusion of the bilateral common carotid arteries (2-VO) from day 7 to day 35 as previously described $(18,19)$. Rats were anesthetized with an intraperitoneal injection $(0.15 \mathrm{ml} / 100 \mathrm{~g})$ of $100 \mathrm{mg} / \mathrm{kg}$ ketamine and $10 \mathrm{mg} / \mathrm{kg}$ xylazine. Subsequently, rats were placed on a stereotactic device. The bilateral common carotid arteries were separated and permanently ligated with small diameter nylon sutures. Rats in the control group underwent the same procedure, but the common carotid arteries were not ligated. The body temperature of the rats was monitored during the whole process and maintained at $37^{\circ} \mathrm{C}$. After surgery, rats were returned to the cages and fed normally.

To determine the effects of miR-150 on cognitive function and hippocampal neurons in VD model rats, rats were subjected to intracerebroventricular (ICV) injections of miR-150 antagomiR (5'-CCCCUCUGGUCAACCAGUCACA-3') or antagomiR negative control (NC, 5'-TTCTCCGAACGTGTCACGT-3') from day 36 to day 38. Both sequences were from Guangzhou RiboBio Co., Ltd. Rats were divided into the following groups ( $\mathrm{n}=12$ per group): i) Control; ii) VD model; iii) antagomiR $\mathrm{NC}$ (treated with 2-VO and antagomiR NC); and iv) miR-150 antagomiR (treated with 2-VO and miR-150 antagomiR). Rats received one ICV injection a day for 3 consecutive days. Rats in the miR-150 antagomiR group received an injection of $200 \mathrm{pmol}$ miR-150 antagomiR dissolved in $5 \mu \mathrm{l}$ sterile double distilled water $\left(\mathrm{ddH}_{2} \mathrm{O}\right)$. Rats in the antaogmiR NC group received an

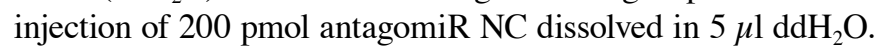
ICV injections were administered through both sides of the lateral ventricle. Based on the rat brain atlas, two small holes were drilled into both sides of the skull using surgical drills. Stereotaxic coordinates for the ICV injection were as follows: Anterior, $-0.8 \mathrm{~mm}$; lateral, $1.5 \mathrm{~mm}$; and depth, $-4.5 \mathrm{~mm}$.

Morris water maze (MWM) test. A water maze with a black circular pool (diameter, $2 \mathrm{~m}$ ) was filled with opaque water by adding black edible pigments and maintained at $25 \pm 1^{\circ} \mathrm{C}$. A diving escape platform (diameter, $20 \mathrm{~cm}$; depth below water, $2.0 \mathrm{~cm}$ ) was located in the center of the first quadrant. Before training, a pupillary light reflex test was performed in all rats. Rats with impaired pupillary light reflex were excluded from the experiment to prevent visual factors affecting the test. Behavioral training consisting of three trials per day for 5 days was conducted. For each behavioral training trial, rats were placed in the water facing the lateral wall and each rat was allowed to find the platform within $120 \mathrm{sec}$. If the platform was not found within $120 \mathrm{sec}$, they were guided to the platform and allowed to rest for at least $20 \mathrm{sec}$ (the escape latency was set to $120 \mathrm{sec}$ in this situation). The DigBehav-Morris water maze video analysis system (Mobile Benchmark Software Technology Co., Ltd.) was used to monitor escape latencies. At the end of the experiment, rats were euthanized with an intraperitoneal injection of $150 \mathrm{mg} / \mathrm{kg}$ sodium pentobarbital. Following euthanasia, animal death was confirmed by observing the lack of heartbeat, respiratory arrest, pupil dilation, and lack of nerve reflex.

Reverse transcription-quantitative PCR (RT-qPCR). Target gene expression levels were measured via RT-qPCR. Total RNA was extracted from rat hippocampus tissues and cells using the PureLink RNA mini kit (Thermo Fisher Scientific, Inc.). Total RNA was reverse transcribed into cDNA using TransScript ${ }^{\circledR}$ Reverse Transcriptase (cat. no. T101-02; Beijing Transgen Biotech Co., Ltd.), according to the manufacturer's protocol. Subsequently, qPCR was performed using Power SYBR $^{\mathrm{TM}}$ Green PCR Master Mix (cat. no. 4367659; Thermo Fisher Scientific, Inc.) on a LightCycler 96 [Roche Diagnostics (Shanghai) Co., Ltd.]. The reaction system $(20 \mu \mathrm{l})$ consisted of $10 \mu 1$ SYBR premix Extaq, $0.6 \mu 1$ forward and reverse primers, $1 \mu \mathrm{l}$ cDNA template (with 10X-dilution) and $7.8 \mu \mathrm{l}$ deionized water. The conditions for RT-qPCR were as follows: Pre-denaturation at $95^{\circ} \mathrm{C}$ for $10 \mathrm{~min}, 40$ cycles of denaturation at $95^{\circ} \mathrm{C}$ for $5 \mathrm{sec}$, annealing at $60^{\circ} \mathrm{C}$ for $30 \mathrm{sec}$, and extension at $95^{\circ} \mathrm{C}$ for $10 \mathrm{sec}$. The sequences of the primers used for qPCR are presented in Table I. GAPDH and U6 were used as internal controls, the $2^{-\Delta \Delta C q}$ method was used to calculate the expression of miRNA and mRNA (20).

Triphenyltetrazolium chloride (TTC) staining. Briefly, brain tissues were rapidly excised from rats and frozen at $-20^{\circ} \mathrm{C}$ for $5 \mathrm{~min}$. Subsequently, tissues were cut into 5-6 uniform coronal sections $(2 \mathrm{~mm})$, which were water-bathed in $2 \%$ TTC (Sigma-Aldrich; Merck KGaA) at $37^{\circ} \mathrm{C}$ in the dark for $30 \mathrm{~min}$. Following fixation with $4 \%$ phosphate-buffered formalin at room temperature for $30 \mathrm{~min}$, sections were observed under light microscope (CX33; Olympus Corporation) and photographed directly with a camera. The infarct area was analyzed using ImageJ software (V1.46, National Institutes of Health).

Immunohistochemistry $(I H C)$. The extracted rat brain tissues were fixed with $1 \%$ paraformaldehyde at room temperature for 30 min, hydrated with 50, 70, 80, 95 and $100 \%$ ethanol for $30 \mathrm{~min}$ each, followed by blocking in xylene containing $50 \%$ ethanol for $2 \mathrm{~h}$, paraffin-embedding, and sectioning $(5 \mu \mathrm{m})$. Paraffin-embedded sections were dewaxed twice with xylene for $10 \mathrm{~min}$ each and rehydrated with gradient 
Table I. Sequences of primers used for reverse transcriptionquantitative PCR.

\begin{tabular}{ll}
\hline Gene & \multicolumn{1}{c}{ Sequence $\left(5^{\prime} \rightarrow 3^{\prime}\right)$} \\
\hline miR-150 & F: CTCCCAACCCTTGTACCA \\
& R: GAACATGTCTGCGTATCTC \\
HOXA1 & F: AGCCACCAAGAAGCCTGTCGTT \\
& R: TTGACCCACGTAGCCGTACTCT \\
U6 & F: CTCGCTTCGGCAGCACAT \\
& R: TTTGCGTGTCATCCTTGCG \\
GAPDH & F: CATCACTGCCACCCAGAAGACTG \\
& R: ATGCCAGTGAGCTTCCCGTTCAG \\
Apaf1 & F: CACGAGTTCGTGGCATATAGGC \\
& R: GGAAATGGCTGTCGTCCAAGGA \\
p53 & F: CCTCAGCATCTTATCCGAGTGG \\
& R: TGGATGGTGGTACAGTCAGAGC \\
Cyto C & F: GAGGCAAGCATAAGACTGGACC \\
& R: ACTCCATCAGGGTATCCTCTCC
\end{tabular}

F, forward; R, reverse; miR, microRNA; HOXA1, homeobox A1; Apaf1, apoptotic peptidase activating factor 1; Cyto C, Cytochrome C.

ethanol (95, 70 and 50\%). After washing under running water for $10 \mathrm{sec}$, the sections were treated with $0.03 \%$ hydrogen peroxide-methanol solution for $10 \mathrm{~min}$ and then heated in a microwave oven for $10 \mathrm{~min}$ after adding $0.01 \mathrm{M}$ citrate buffer (pH 6.0). Subsequently, tissue sections were sealed with $10 \%$ bovine serum albumin at room temperate for $30 \mathrm{~min}$ and incubated with anti-c-fos (cat. no. ab222669; 1:2,000; Abcam) and anti-HOXA1 (cat. no. ab230513; 1:5,000; Abcam) primary antibodies overnight at $4^{\circ} \mathrm{C}$. Following primary incubation, tissue sections were incubated with the secondary goat anti-rabbit IgG (cat. no. ab6721; 1:1,000; Abcam) or goat anti-rat IgG (cat. no. ab6734; 1:1,000; Abcam) for $30 \mathrm{~min}$ at room temperature. Following thorough washing with PBS, chromogen detection was performed using diaminobenzidine (Beijing Solarbio Science \& Technology Co., Ltd.). All sections were scanned using a Panoramic MIDI high-resolution pathology slice scanner (3DHistech, Ltd.) and analyzed using ImageJ with FIJI installed (National Institutes of Health). To calculate the positive cell rate, the area of density (AOD) was calculated according to the following formula: $\mathrm{AOD}=$ integrated optical density/total area.

Nissl staining. Hippocampal neurons were observed by performing Nissl staining according to a previously described protocol (21). Sections were fixed with $1 \%$ paraformaldehyde at room temperature for $30 \mathrm{~min}$, rehydrated in distilled water and stained with $0.5 \%$ crystal violet solution for $10 \mathrm{~min}$. Brain sections were imaged using a DM5000B bright-field microscope (magnification, x400; Leica Microsystems GmbH). Normal neurons with visible nuclei in the CA1 subdomain of the hippocampal region were circled, counted within an unbiased counting field $(200 \times 130 \mu \mathrm{m})$ in each group and expressed as the number of cells $/ \mathrm{mm}^{2}$ in the CA1 region of the hippocampus. The number of neurons was assessed in five brain sections from each rat at different depths on the left and right sides.

Hematoxylin and eosin $(H \& E)$ staining. Brain tissues were fixed with $1 \%$ paraformaldehyde at room temperature for $30 \mathrm{~min}$, embedded in paraffin, cut into $5-\mu \mathrm{m}$ thick sections and heated at $60^{\circ} \mathrm{C}$ for $25 \mathrm{~min}$. After rehydration using graded alcohols (95, 70 and 50\%), tissue sections were rinsed in distilled water, stained using in $0.5 \%$ hematoxylin solution for 15 min at room temperature, then washed, and stained with eosin solution in 95\% ethanol for 3-5 $\mathrm{min}$ at room temperature. Pathological alterations to neurons in the CA1 region of the hippocampus were observed using an optical microscope (Olympus Corporation). Scores of post 2VO surgery-related injuries were observed under a light microscope (CX33, Olympus Corporation) at x200 magnification in five brain regions per rat were evaluated. A damage score based on the system proposed by Vannucci et al (22) was used to assess the tissue sections: 0 , no damage; 1 , focal loss of a single neuron; 2 , multiple damage in the hippocampus; 3 , multiple or large foci of neuronal loss with edema and reactive glial hyperplasia, but without cystic infarction; and 4, severe cystic and non-cystic infarction, edema, myelin pallor and reactive glial hyperplasia.

TUNEL staining. The apoptotic index of rat hippocampal tissues was evaluated using the TUNEL apoptosis detection kit (Roche Diagnostics) according to the manufacturer's protocol. Briefly, after being fixed with $1 \%$ paraformaldehyde at room temperature for $30 \mathrm{~min}$, paraffin-embedded sections were dewaxed and rehydrated in a gradient ethanol $(95,70$ and $50 \%$ ). Subsequently, tissue sections were detached with trypsin at room temperature for $40 \mathrm{~min}$. Tissue sections were then incubated with TUNEL reaction buffer at $37^{\circ} \mathrm{C}$ for $1 \mathrm{~h}$ in humidity (45-65\%). The sections were immersed in $0.5 \%$ hematoxylin solution for $15 \mathrm{~min}$ at room temperature for the nuclear staining. Following washing with PBS, TUNEL-positive cells and normal cells in each group were observed under five fields of view using a light microscope (magnification x200; CX33; Olympus Corporation).

Quantification of lactic dehydrogenase ( $L D H)$ content and Caspase-3 activity. The level of LDH was determined using a cytotoxic LDH assay kit (Dojindo Molecular Technologies, Inc.) according to the manufacturer's protocol. Briefly, the tissues were ground on ice with TRIzol reagent (Invitrogen; Thermo Fisher Scientific, Inc.) at a volume ratio of 1:10, followed by centrifugation at $161,2.8 \times \mathrm{g}$ to extract the supernatant. The brain tissue lysate was plated into 96 -well plates at $10 \mu \mathrm{l}$ per well, and $100 \mu \mathrm{l}$ working solution was added to each well. Following incubation for $30 \mathrm{~min}$ in the dark at room temperature, $50 \mu 1$ termination solution was added. The level of LDH was assessed by measuring the absorbance at a wavelength of $490 \mathrm{~nm}$ using a microplate reader. $\mathrm{LDH}$ activity unit $(\mathrm{mU} / \mathrm{ml})$ was calculated according to the following formula: [optical density (OD) value sample $^{-O D}$ value $\left._{\text {blank control }}\right] /(\mathrm{OD}$ value standard tube $\mathrm{OD}$ value standard blank control tube) x standard concentration.

The brain tissue lysate was prepared as described above. Caspase- 3 activity in rat brain tissue lysate was assessed using the Caspase-Glo ${ }^{\circledR} 3$ assay (cat. no. G8981; Promega 
Corporation), a luminescence-based test system that measures caspase-3 activity, according to the manufacturer's protocol. The luminescence signal was quantified using a microplate fluorometer at $405 \mathrm{~nm}$.

Cell culture. PC12 cells (Procell Life Science \& Technology Co., Ltd.) were cultured in RPMI-1640 (Gibco; Thermo Fisher Scientific, Inc.) supplemented with $10 \%$ FBS (Gibco; Thermo Fisher Scientific, Inc.) and $1 \%$ penicillin-streptomycin solution (Thermo Fisher Scientific, Inc.) at $37^{\circ} \mathrm{C}$ with $5 \% \mathrm{CO}_{2}$. Cell culture medium was renewed every 3 days.

PC12 cells were seeded $\left(5 \times 10^{6}\right.$ cells/well) in 6 -well plates with complete medium. At $80 \%$ confluence, cells were transfected with miR-150 mimic, mimic negative control (NC), miR-150 inhibitor, inhibitor NC, miR-150 mimic + empty vector, miR-150 mimic + HOXA1, miR-150 inhibitor + small interfering RNA (si) Scramble (Scr), miR-150 inhibitor + si-HOXA1-\#1 or miR-150 inhibitor + si-HOXA1-\#2 (the final concentration was $50 \mathrm{ng} / \mu \mathrm{l})$. The following sequences were used: miR-150 mimic, 5'-CCCCUCUGGUCAACCAGU CACA-3'; miR-150 inhibitor, 5'-AGAGGGTTGGGAACA TGGTCAC-3'; NC for both miR-150 mimic and inhibitor, 5'-TCTCCCAACCCTTGTCCAGTG-3'; si-HOXA1-\#1, CCG GCCCTCGGACCATAGGATTACACTCGAGTGTAATCC TATGGTCCGAGGGTTTTTG; si-HOXA1-\#2, CCGGCC GCTGTTTACTCTGGAAATCCTCGAGGATTTCCAGAG TAAACAGCGGTTTTTG; si-Scr, GGCCGGGAGCCT AGGTATCCTAATGGTACGAGGCTAGCTGGGATC; and HOXA1, 5'-TGGCTTTTGAAGGGAGTTCTGATTTTT CTTCTCCGGCCCCA-3'. For transfection, miR-mimics, miR-inhibitors, siRNAs and vectors were centrifuged at $447.2 \mathrm{x} \mathrm{g}$ for $3 \mathrm{~min}$ at $4^{\circ} \mathrm{C}$, and then dissolved in $125 \mu \mathrm{l}$ diethyl pyrocarbonate water. Subsequently, $4 \mu \mathrm{l}$ miR-mimic, miR-inhibitor, siRNA or vector solution was mixed with $7.5 \mu 1$ Lipofectamine ${ }^{\circledR} 2000$ (Invitrogen; Thermo Fisher Scientific, Inc.), incubated for $5 \mathrm{~min}$ at room temperature, allowed to stand for $20 \mathrm{~min}$ at room temperature, and then added to each well of the 6-well plate. After $48 \mathrm{~h}$ of transfection, the medium was changed to medium supplemented with $10 \%$ FBS and antibiotics, followed by another incubation for $48 \mathrm{~h}$. At $70 \%$ confluence, cells were passaged and cultured. Stable cell lines were screened using $10 \mu \mathrm{g} / \mathrm{ml}$ puromycin or $250 \mu \mathrm{g} / \mathrm{ml}$ G418. Following a 2-week maintenance $(5 \mu \mathrm{g} / \mathrm{ml}$ puromycin or $200 \mu \mathrm{g} / \mathrm{ml} \mathrm{G} 418)$, miR-150 or HOXA1 expression levels were detected via RT-qPCR and western blotting, respectively.

PI/Hoechst 33342 staining. PI/Hoechst 33342 staining was performed according to the manufacturer's protocol. PC12 cells were seeded at $5 \times 10^{3}$ cells/well and incubated with both $10 \mu \mathrm{g} / \mathrm{ml}$ Hoechst 33342 (cat. no. C1017; Beyotime Institute of Biotechnology) and $10 \mu \mathrm{g} / \mathrm{ml}$ PI (cat. no. ST512; Beyotime Institute of Biotechnology) at $37^{\circ} \mathrm{C}$ for $15 \mathrm{~min}$, respectively. Stained cells were observed in five randomly selected fields of view using a fluorescence microscope to calculate the number of apoptotic cells using ImageJ software (V1.46; National Institutes of Health).

Flow cytometry. Cell apoptosis was detected via flow cytometry using the Annexin V/PI kit (BD Biosciences). Briefly, PC12 cells were seeded at $5 \times 10^{3}$ cells/well and resuspended in
$1 \mathrm{X}$ binding buffer. Subsequently, $\mathrm{PC} 12$ cells were stained with Annexin $\mathrm{V}$ and $\mathrm{PI}$ at $37^{\circ} \mathrm{C}$ for $30 \mathrm{~min}$ in a dark room. FACS analysis was performed using a FACSCalibur flow cytometer (BD Biosciences) using FlowJo V10.6.2 (BD Biosciences). Early apoptotic cells are in the lower right quadrant, while late apoptotic cells in the upper right quadrant.

Western blotting. Total protein was isolated from brain tissues and PC12 cells using RIPA assay lysis buffer (cat. no. P0013C; Beyotime Institute of Biotechnology) on ice. Total protein was quantified using the BCA method. Proteins $(20 \mu \mathrm{g})$ were separated via 10\% SDS-PAGE and transferred onto PVDF membranes (EMD Millipore). Following blocking with 5\% skimmed milk for $2 \mathrm{~h}$ at $37^{\circ} \mathrm{C}$, the membranes were incubated overnight at $4^{\circ} \mathrm{C}$ with primary antibodies targeted against: HOXA1 (cat. no. ab230513; 1:5,000; Abcam), apoptotic peptidase activating factor 1 (Apaf1; cat. no. 8969; 1:2,000; Cell Signaling Technology, Inc.), p53 (cat. no. 2524; 1:5,000; Cell Signaling Technology, Inc.), Cytochrome C (Cyto C; cat. no. 11940; 1:2,000; Cell Signaling Technology, Inc.) and GAPDH (cat. no. ab8245; 1:5,000; Abcam). Subsequently, the membranes were incubated with HRP-conjugated secondary antibody against IgG (cat. no. ab6721; 1:10,000; Abcam) for $2 \mathrm{~h}$ at $37^{\circ} \mathrm{C}$. Following washing with TBS with $0.1 \%$ Tween-20, protein bands were visualized using an enhanced chemiluminescence detection system (Thermo Fisher Scientific, Inc.). Semi-quantification was conducted using Gel-Pro Analyzer version 4.0 (Media Cybernetics, Inc.).

Dual-luciferase reporter gene assay. PicTar (https://pictar. mdc-berlin.de/) was used to verify whether HOXA1 was the direct target gene of miR-150. Dual-luciferase reporter assays were performed to verify the interaction between miR-150 and the 3'-untranslated region (UTR) of HOXA1. The wild-type (WT) HOXA1 sequence from HOXA1 mRNA (including the predicted binding sites of miR-150) was amplified and inserted into the pLUC dual-luciferase vector (Promega Corporation) to construct the pmirGLO-HOXA1-WT reporter vector. The presumed miR-150 binding site in HOXA1 3'-UTR was mutated using GeneArt TM site-directed mutagenesis PLUS system (cat. no. A14604; Thermo Fisher Scientific, Inc.). The mutant type (MUT)HOXA1 3'-UTR was inserted into the pLUC vector to develop the pLUC-HOXA1-MUT reporter vector. 293T cells (American Type Culture Collection) were seeded into a 96-well plate at $5 \times 10^{3}$ cells/well and co-transfected with $50 \mathrm{ng}$ pmirGLO-HOXA1-WT or pLUC-HOXA1-MUT and 50 pmol miR-150 mimic (5'-CCCCUCUGGUCAACCAGUCACA-3') or NC mimic (5'-TTCTCCGAACGTGTCACGT-3', both from Guangzhou RiboBio Co., Ltd.). Transfection was performed at $37^{\circ} \mathrm{C}$ for $24 \mathrm{~h}$ using Lipofectamine ${ }^{\circledR} 2000$ reagent (Invitrogen; Thermo Fisher Scientific, Inc.). At 48 h post-transfection, luciferase activities were measured using the Dual-Luciferase Reporter Assay System (Promega Corporation) and the firefly luciferase activity value was compared against the Renilla luciferase activity value.

Statistical analysis. Statistical analyses were performed using SPSS software (version 22.0; IBM Corp.). Data are presented as the mean \pm SD. To assess the normality of the data, the Kolmogorov-Smirnov test was used. Comparisons between 
A

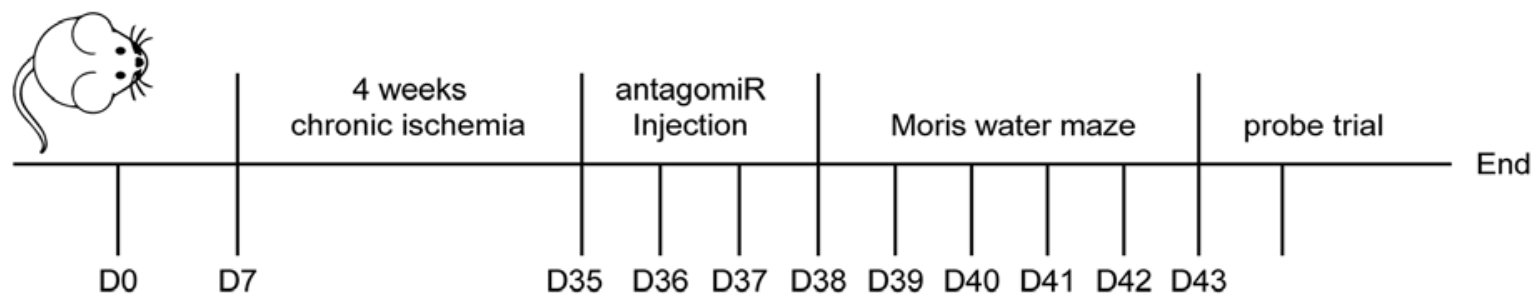

B

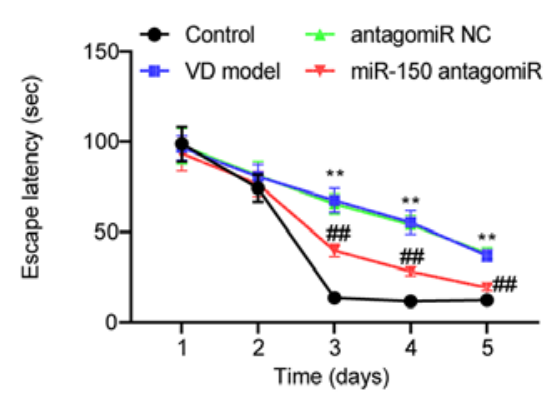

C

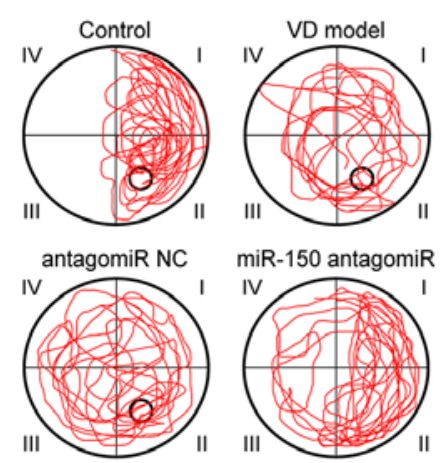

D

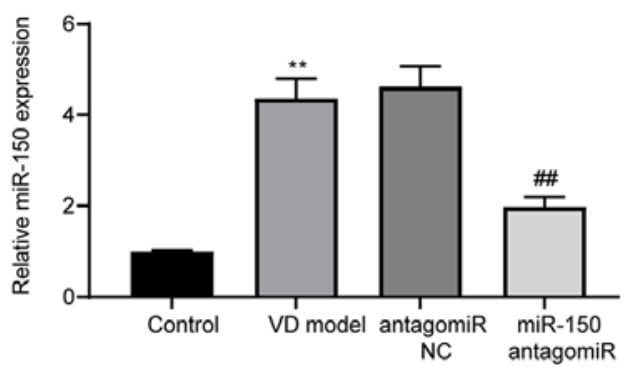

Figure 1. miR-150 antagomiR treatment enhances memory and learning abilities in VD model rats. (A) Timeline of rat experiments (n=12 per group). (B) Escape latency of rats during the first 5 days using the MWM test. (C) Representative movement tracking of rats on the 5th day as measured using the MWM test. (D) miR-150 expression levels in the brain tissues of rats were determined via reverse transcription-quantitative PCR. Experiments were repeated three times. Data are presented as the mean \pm standard deviation. Two-way mixed ANOVA followed by Tukey's post hoc test was used to analyze the data presented (B). One-way ANOVA followed by Tukey's post hoc test was used to analyze the data presented (D). ${ }^{* *} \mathrm{P}<0.01$ vs. control; ${ }^{\# \prime} \mathrm{P}<0.01 \mathrm{vs}$. antagomiR NC. miR, microRNA; VD, vascular dementia; MWM, Morris water maze; NC, negative control; D, day.

two groups were analyzed using an unpaired Student's t-test. Comparisons among multiple groups were analyzed using one-way ANOVA or two-way mixed ANOVA followed by Tukey's post hoc test. All experiments were repeated three times. $\mathrm{P}<0.05$ was considered to indicate a statistically significant difference.

\section{Results}

miR-150 knockdown strengthens the learning and memorial abilities of VD model rats. VD model rats were established by performing 2-VO surgery. After 35 days, rats were injected with miR-150 antagomiR or antagomiR NC. To verify whether miR-150 altered the spatial learning and memory abilities of VD model rats, the MWM test was performed at 3 days post-ICV injection (Fig. 1A). During MWM training, the escape latency of rats was notably reduced in a time-dependent manner, indicating that the platform could be found with training (Fig. 1B). Compared with the control group, the escape latency of the rats in the VD model group was significantly increased. By contrast, the escape latency of the rats in the miR-150 antagomiR group was significantly shorter compared with the antagomiR NC model group.

On the 5th day of the MWM test, the number of VD model rats crossing the platform was notably decreased compared with the control group; however, rats treated with miR-150 antagomiR displayed the opposite results (Fig. 1C). miR-150 expression levels in rat brain tissues were measured (Fig. 1D). Compared with the control group, miR-150 expression levels were significantly increased in the brain tissues of VD model rats. miR-150 expression was significantly downregulated by miR-150 antagomiR treatment compared with the antagomiR
NC group. As indicated by the MWM test, spatial learning abilities were impaired when rats were exposed to 4 -week chronic ischemia. Moreover, miR-150 knockdown alleviated cognitive dysfunction, and enhanced learning and memory abilities in VD model rats.

miR-150 knockdown improves hippocampal neuron activity. Subsequently, brain tissues were extracted from rats for histological staining and detection of protein expression. The infarct area in brain tissues was measured by performing TTC staining. The infarct area was significantly increased in the brain tissues of VD model rats compared with control rats, whereas miR-150 knockdown in brain tissues significantly reduced 2-VO operation-induced cerebral infarction compared with the antagomiR NC group (Fig. 2A). Moreover, IHC was conducted to detect the expression of c-fos protein in hippocampal tissues of the CA1 region in brain tissues (Fig. 2B). The IHC results demonstrated that $\mathrm{c}$-fos protein expression was significantly reduced in the hippocampal tissues of VD model rats compared with control rats; however, miR-150 knockdown significantly reversed VD-induced downregulation of c-fos protein expression compared with the antagomiR NC group. Subsequently, Nissl staining was performed to assess the number of Nissl bodies in rat hippocampal tissues to measure hippocampal neuron activity. Compared with the control group, the VD model group displayed significantly suppressed rat hippocampal neuron activity, which was restored by miR-150 antagomiR treatment (Fig. 2C).

miR-150 knockdown suppresses hippocampal neuron apoptosis in VD model rats. H\&E staining was performed to detect the pathological structure of rat brain tissues (Fig. 3A). 
A
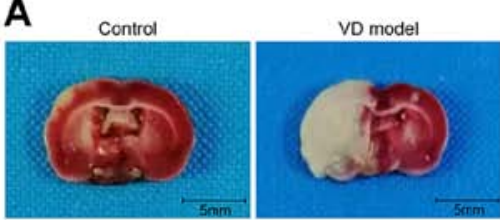

B

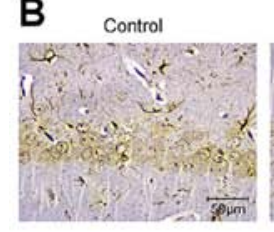

C Control

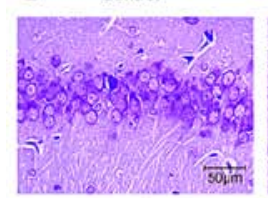

VD model

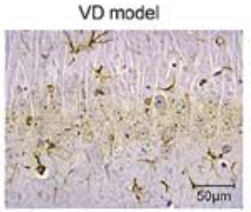

VD model

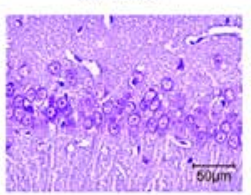

antagomiR NC

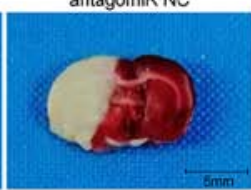

antagomiR NC

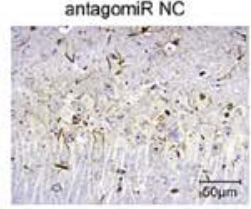

antagomiR NC

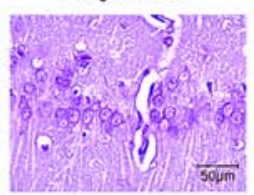

miR-150 antagomiR

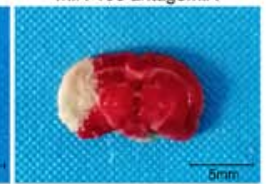

miR-150 antagomiR

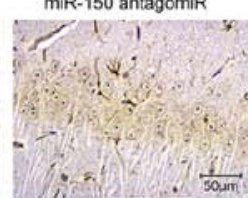

miR-150 antagomiR

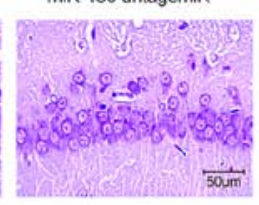

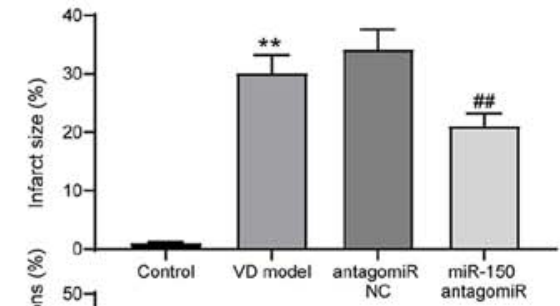
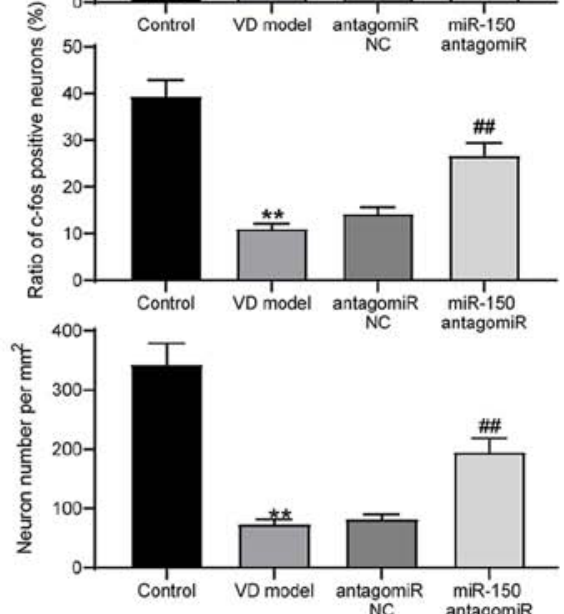

Figure 2. miR-150 antagomiR enhances the activity of hippocampal neurons of rats. (A) Infarction area in the brain tissues of rats as determined via triphenyltetrazolium chloride staining (scale bar, $5 \mathrm{~mm}$ ). (B) c-fos protein expression levels in brain tissues were examined via immunohistochemistry (scale bar, $50 \mu \mathrm{m}$ ). (C) Neuron activity in the brain tissues of rats was measured by performing Nissl staining (scale bar, $50 \mu \mathrm{m}$ ). Experiments were repeated three times. Data are presented as the mean \pm standard deviation. One-way ANOVA followed by Tukey's post hoc test was used to analyze the presented data. ${ }^{* *} \mathrm{P}<0.01$ vs. control; ${ }^{\#} \mathrm{P}<0.01$ vs. antagomiR NC. miR, microRNA; NC, negative control; VD, vascular dementia.

A
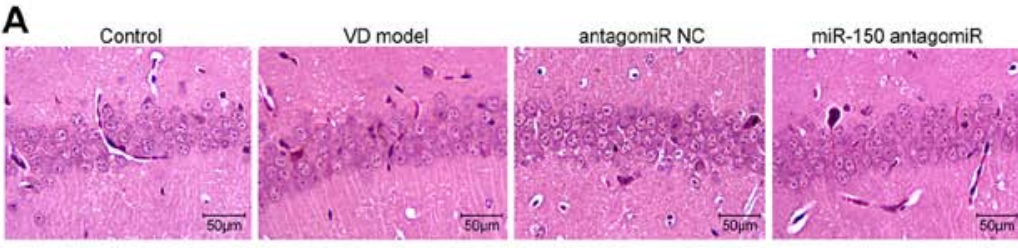

B

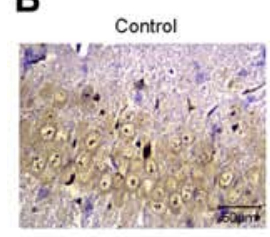

C

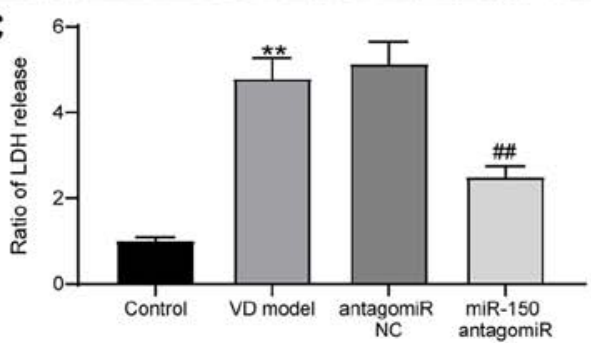

VD model
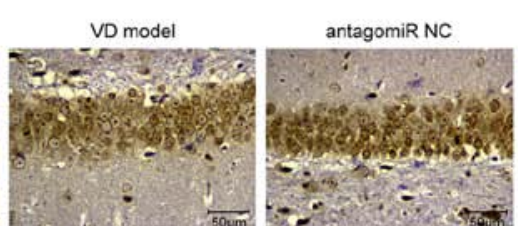

miR-150 antagomiR

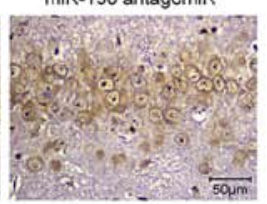

D

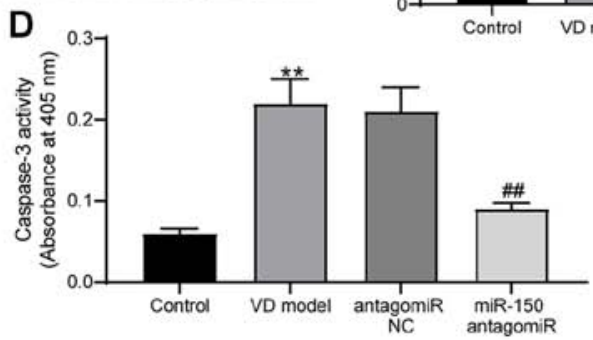

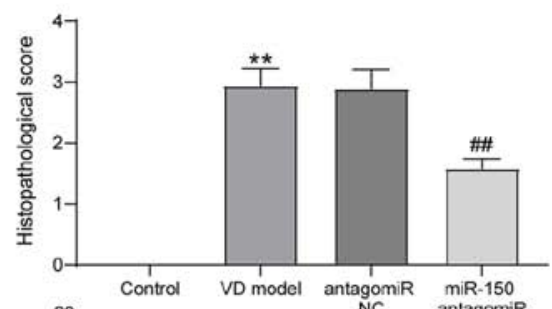

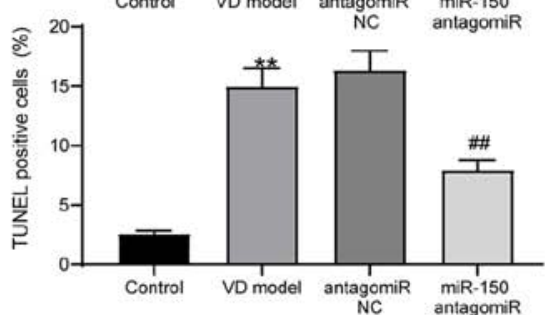

NC miR-150

Figure 3. miR-150 antagomiR suppresses hippocampal neuron apoptosis in rats. (A) Levels of fibrosis in rat brain tissues were determined by performing hematoxylin and eosin staining (scale bar, $50 \mu \mathrm{m}$ ). (B) Apoptosis of hippocampal tissues in rat brain tissues was examined by performing TUNEL staining (scale bar, $50 \mu \mathrm{m}$ ). (C) LDH contents in rat brain tissues were assessed using an LDH kit. (D) Caspase-3 activities in rat brain tissues were detected using a Caspase-3 kit. Experiments were repeated three times. Data are presented as the mean \pm standard deviation. One-way ANOVA followed by Tukey's post hoc test was used to analyze the presented data. ${ }^{* *} \mathrm{P}<0.01$ vs. control; ${ }^{\# \#} \mathrm{P}<0.01$ vs. antagomiR NC. miR, microRNA; LDH, lactic dehydrogenase; NC, negative control; VD, vascular dementia.

Compared with the control group, pathological injury in the brain tissues of VD model rats was significantly increased, which was accompanied by blurred cell boundaries. miR-150 knockdown significantly relieved the level of pathological injury in VD model rats compared with the antagomiR NC group. The TUNEL staining results indicated that miR-150 

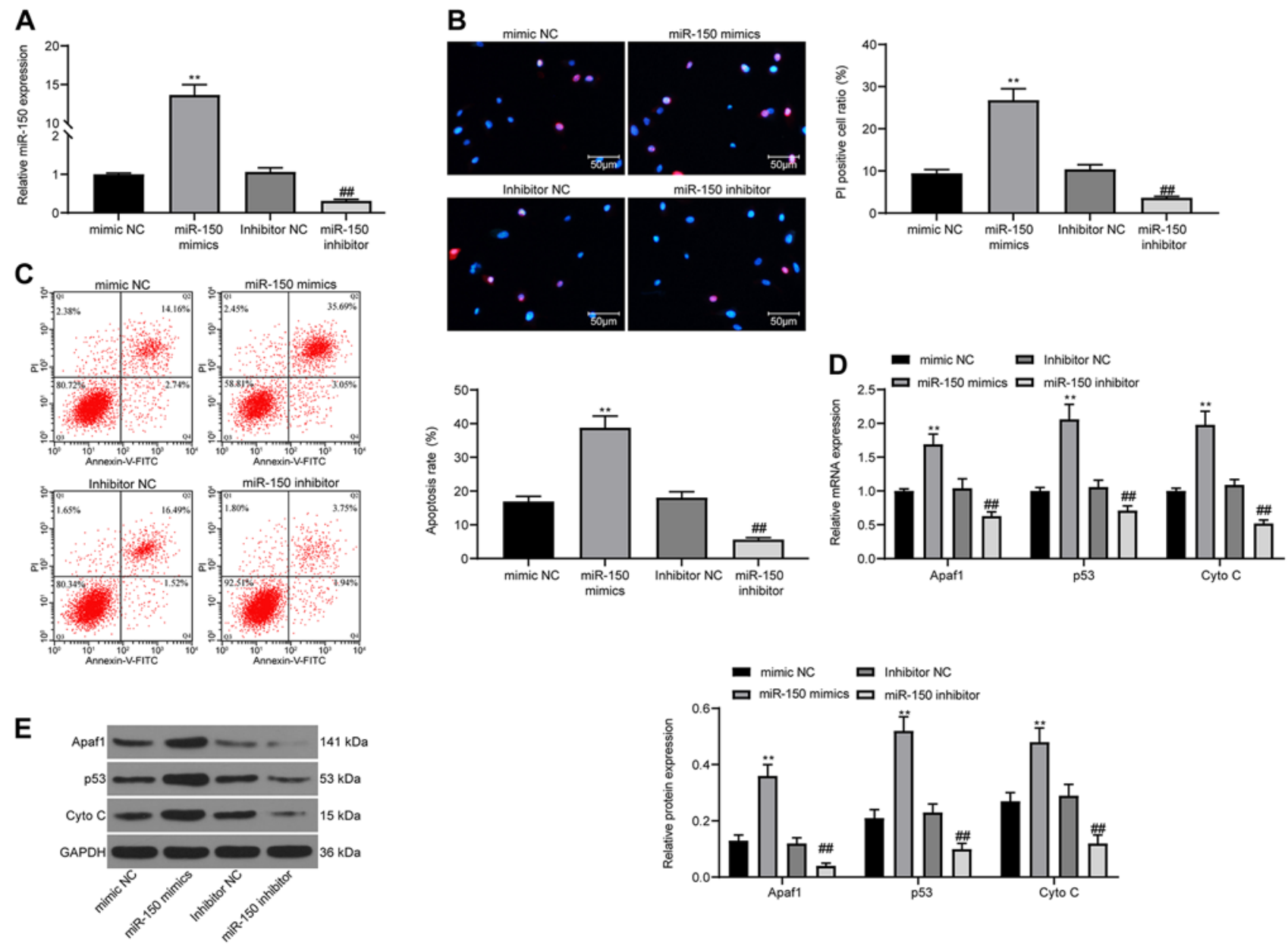

Figure 4. miR-150 mimic promotes PC12 cell apoptosis. (A) Transfection efficiency of miR-150 mimics and miR-150 inhibitor. (B) Cell apoptosis was determined by performing PI/Hoechst 33342 double staining. (C) Apoptotic cells were analyzed via flow cytometry. Apoptosis-related factors Apaf1, p53 and Cyto C (D) mRNA and (E) protein expression levels were determined via reverse transcription-quantitative PCR and western blotting. Experiments were repeated three times. Data are presented as the mean \pm standard deviation. One-way ANOVA followed by Tukey's post hoc test was used to analyze the presented data. ${ }^{* *} \mathrm{P}<0.01$ vs. mimic $\mathrm{NC}$; ${ }^{\# \#} \mathrm{P}<0.01$ vs. inhibitor NC. miR, microRNA; Apaf1, apoptotic peptidase activating factor 1 ; Cyto C, Cytochrome C; $\mathrm{NC}$, negative control.

antagomiR significantly inhibited 2-VO surgery-induced cell apoptosis in hippocampal tissues in the CA1 region compared with the antagomiR NC group (Fig. 3B). Additionally, the content of LDH and activity of Caspase- 3 in rat brain tissues were assessed using LDH and Caspase-3 kits, respectively (Fig. 3C and D). The content of LDH and activity of Caspase-3 in VD model rat brain tissues were significantly increased compared with the control group. miR-150 knockdown significantly inhibited VD-induced alterations to LDH content and Caspase-3 activity compared with the antagomiR NC group.

miR-150 overexpression promotes PC12 cell apoptosis. To validate the role of miR-150 in neurons in vitro, $\mathrm{PC} 12$ cells were used in the present study. miR-150 expression was overexpressed or knocked down in PC12 cells. Transfection efficiencies were assessed via RT-qPCR (Fig. 4A). Subsequently, cell apoptosis was detected via Hoechst 33342/PI staining. The results demonstrated that miR-150 overexpression significantly promoted cell apoptosis compared with the mimic NC group, whereas miR-150 knockdown significantly reduced cell apoptosis compared with the inhibitor NC group (Fig. 4B). The detection of cell apoptosis via flow cytometry displayed the same experimental results (Fig. 4C). Furthermore, RT-qPCR and western blotting were performed to detect the expression levels of apoptosis-related proteins Apaf1, p53 and Cyto C (Fig. 4D and E). miR-150 overexpression significantly increased the expression levels of apoptosis-related proteins compared with the mimic NC group, whereas miR-150 knockdown displayed the opposite effects on protein expression compared with the inhibitor NC group.

miR-150 negatively modulates HOXAl expression via direct binding. To explore the downstream molecular mechanism underlying miR-150, PicTar was used to predict the possible targets of miR-150. PicTar identified a potential binding relationship between miR-150 and HOXA1 (Fig. 5A). To evaluate the direct effect of miR-150 on HOXA1 expression, a dual-luciferase reporter gene pLUC-HOXA1 with an miR-150 target site was constructed (Fig. 5B). miR-150 overexpression significantly decreased the luciferase activity of HOXA1-WT in 293T cells compared with the mimic control group (Fig. 5C). Therefore, western blotting and IHC were performed to detect HOXA1 expression in the hippocampus of the CA1 region in rat brain tissues. HOXA1 expression 
A

\begin{tabular}{|c|c|c|}
\hline Binding Site & Class & Alignment \\
\hline chr7:27222053-27222058[-] & $6 \mathrm{mer}$ & $\begin{array}{r}\text { Target: } 5 \text { ' acacccucccucuacUGGGAGc } \\
1 \text { I I I I I I } \\
\text { miRNA: } 3 \text { ' gugaccauguucccaACCCUCu } \\
\text { ' }\end{array}$ \\
\hline
\end{tabular}

C

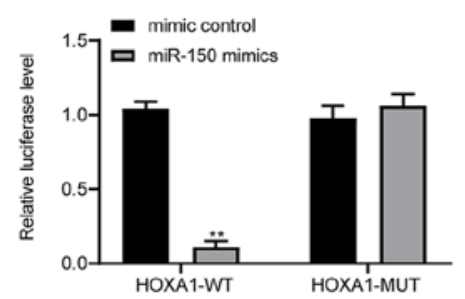

E

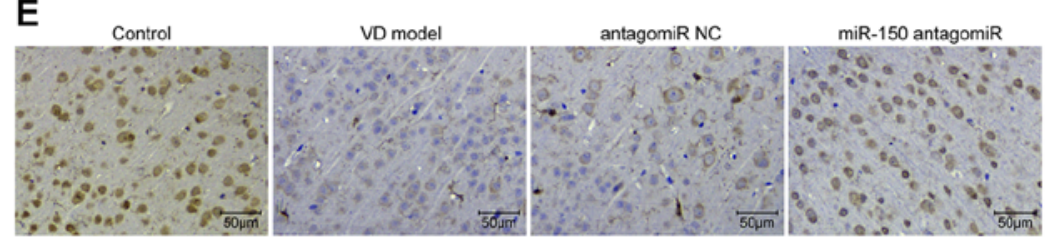

F

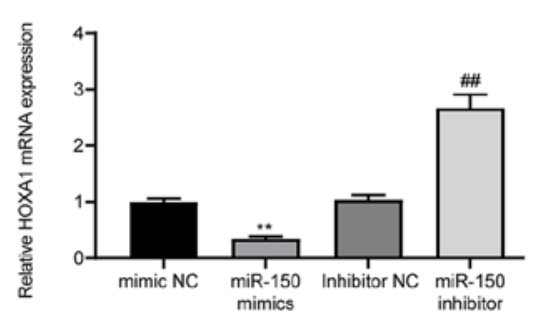

\section{D}

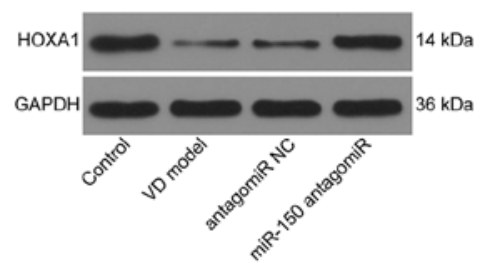

miR-150 antagomiR
G

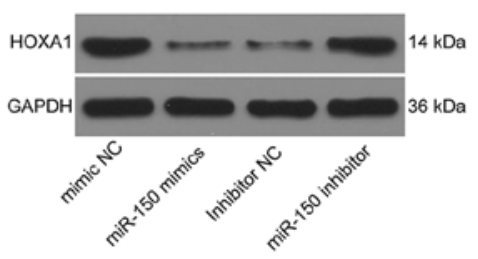

B

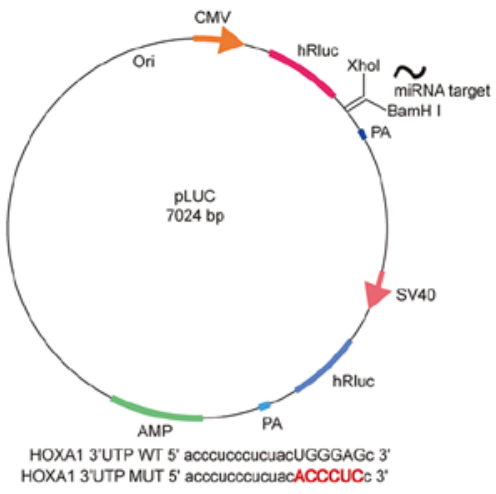

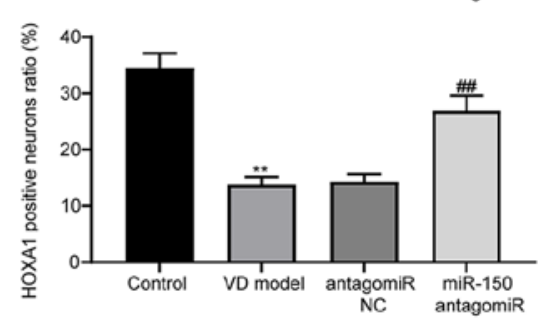

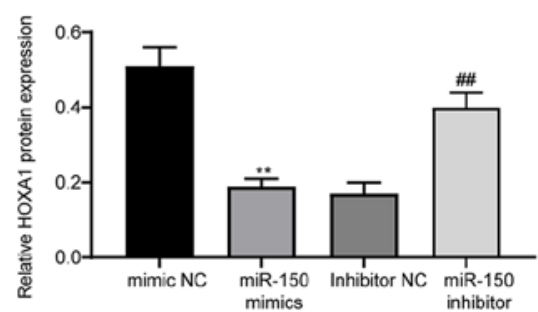

Figure 5. miR-150 negatively regulates HOXA1 expression. (A) PicTar predicted the binding site between HOXA1 3'UTR and miR-150. (B) Establishment of pLUC-HOXA1-MUT/WT reporter vectors containing miR-150 target sites. (C) Dual-luciferase reporter gene assays were determined to assess the interaction between miR-150 and HOXA1. (D) HOXA1 protein expression levels in rat brain tissues were determined via western blotting. (E) HOXA1 positive cells in rat brain tissues were observed via immunohistochemistry. HOXA1 (F) mRNA and (G) protein expression levels in PC12 cells were determined via reverse transcription-quantitative PCR and western blotting, respectively. Experiments were repeated three times. Data are presented as the mean \pm standard deviation. An unpaired Student's t-test was used to analyze the data presented (C). One-way ANOVA followed by Tukey's post hoc test was used to analyze the data presented (D-G). ${ }^{* *} \mathrm{P}<0.01$ vs. mimic control, control or mimic NC; ${ }^{\# P} \mathrm{P}<0.01$ vs. antagomiR NC or inhibitor NC. miR, microRNA; HOXA1, homeobox A1; UTR, untranslated region; MUT, mutant; WT, wild-type; NC, negative control; CMV, Cytomegalovirus; VD, vascular dementia.

in VD model rats was significantly reduced compared with control rats, and VD-induced downregulation of HOXA1 expression was significantly reversed by $\mathrm{miR}-150$ antagomiR treatment compared with the antagomiR NC group (Fig. 5D and E). HOXA1 expression in PC12 cells was further analyzed via RT-qPCR and western blotting. miR-150 overexpression significantly decreased HOXA1 expression compared with the mimic NC group, whereas miR-150 knockdown significantly increased HOXA1 expression compared with the inhibitor NC group (Fig. 5F and G).

HOXA1 overexpression suppresses miR-150-induced PC12 cell apoptosis. Based on the aforementioned results, HOXA1 was overexpressed in miR-150-overexpression PC12 cells, but knocked down in miR-150-knockdown PC12 cells. Western blotting was performed to assess the transfection efficiency of HOXA1 overexpression and knockdown (Fig. 6A). HOXA1 overexpression significantly increased HOXA1 expression levels compared with cells transfected with empty vector. HOXA1 expression in cells transfected with siHOXA1-\#1 or siHOXA1-\#2 was significantly lower compared with cells transfected with Scr. The aforementioned results demonstrated successful transfection of the HOXA1 overexpression vector, siHOXA1-\#1 and si-HOXA1\#2. The Hoechst 33342/PI double staining and flow cytometry results demonstrated that compared with the empty vector group, HOXA1 overexpression significantly inhibited cell apoptosis in miR-150-overexpression PC12 cells (Fig. 6B and C). By contrast, compared with 
A
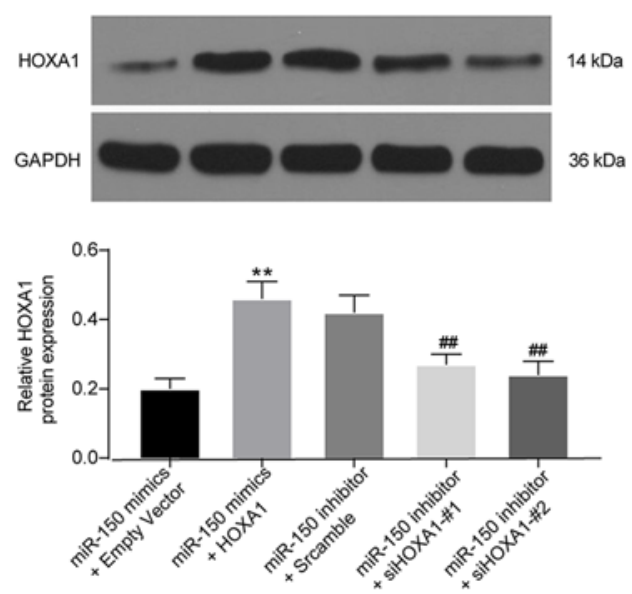
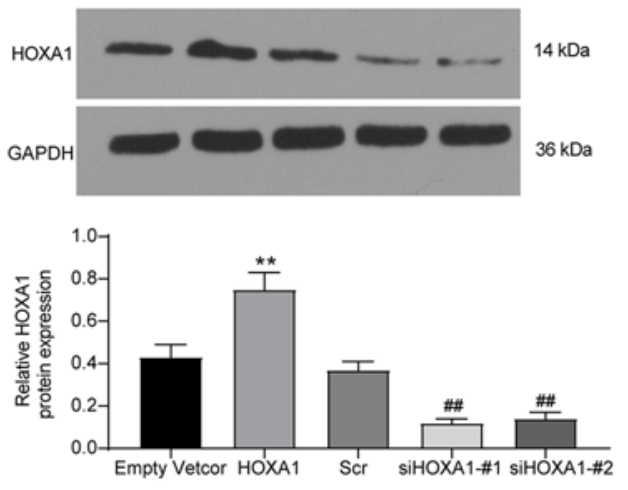

B
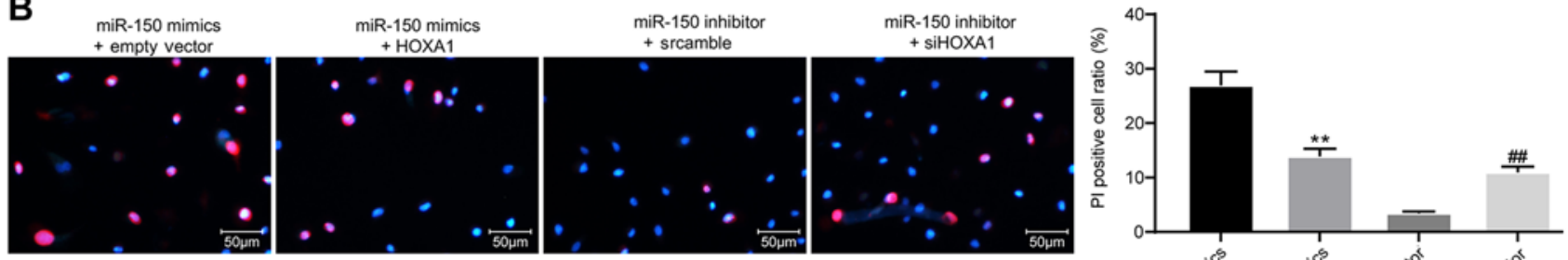

C
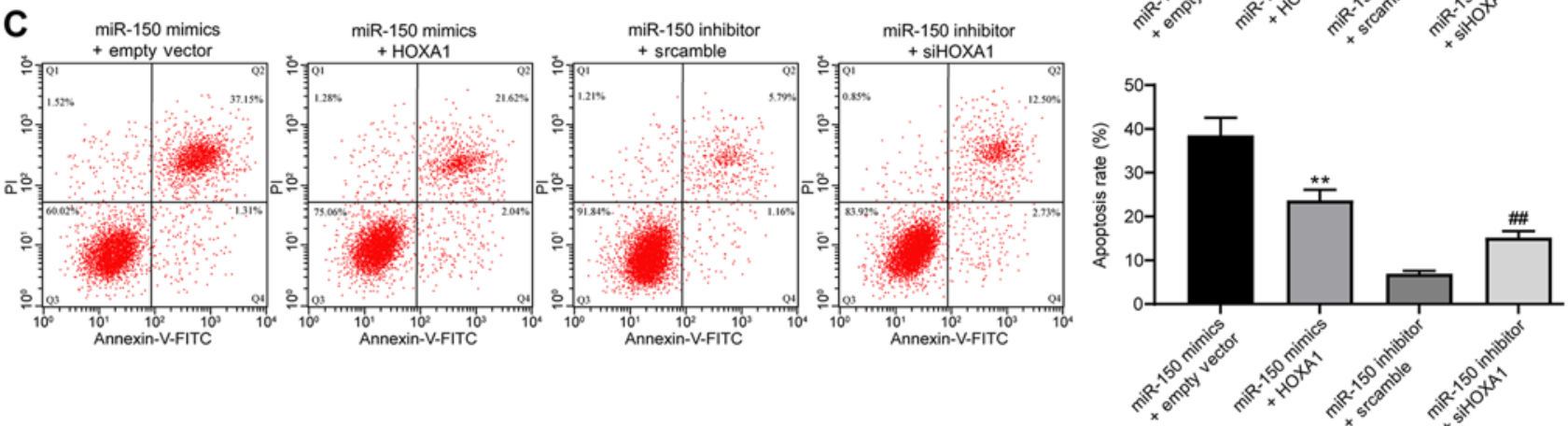

Figure 6. HOXA1 overexpression suppresses miR-150-induced PC12 cell apoptosis. (A) Transfection efficiency of HOXA1 overexpression and knockdown. (B) Cell apoptosis was determined by performing PI/Hoechst 33342 double staining. (C) Apoptotic cells were analyzed via flow cytometry. Experiments were repeated three times. Data are presented as the mean \pm standard deviation. One-way ANOVA followed by Tukey's post hoc test was used to analyze the presented data. ${ }^{* *} \mathrm{P}<0.01$ vs. miR-150 + empty vector; ${ }^{\#} \mathrm{P}<0.01$ vs. miR-150 inhibitor + scramble. HOXA1, homeobox A1; miR, microRNA; si, small interfering RNA.

the Scr group, HOXA1 knockdown significantly promoted miR-150-knockdown PC12 cell apoptosis.

\section{Discussion}

The present study investigated whether miR-150 knockdown displayed an inhibitory effect on VD via targeting HOXA1 in vivo and in vitro. VD model rats were established using 2-VO methods. PC12 cells are also often used as a cell model for neuronal studies (23), thus PC12 cells were used in the present study. The results suggested that chronic ischemia-induced VD model rats displayed learning disorders after 4 weeks, which implied that the VD rat model was successfully established.

Initially, the effects of miR-150 on cognitive function and hippocampal neurons in VD model rats were explored. VD model rats were subjected to ICV injections of miR-150 antagomiR, followed by the detection of cognitive functions by performing MWM tests. miR-150 antagomiR significantly shortened the escape latency of VD model rats compared with the antagomiR NC group. A previous study reported that escape latency is usually prolonged following VD modeling (24). Therefore, miR-150 knockdown should improve cognitive function in VD model rats. Furthermore, compared with antagomiR NC, miR-150 antagomiR elevated c-fos protein expression levels and increased the number of Nissl bodies in the brain tissue of VD model rats. C-fos is aberrantly expressed during neuronal activity processes and an infarction is considered as a biomarker for VD (25). Furthermore, ischemic model rats display a reduced density of Nissl bodies in neurons (26). Collectively, the results of the present study demonstrated that compared with the antagomiR NC group, miR-150 knockdown enhanced the activity of hippocampal neurons. Furthermore, compared with the antagomiR NC group, miR-150 knockdown significantly reduced fibrosis levels, LDH contents and Caspase-3 activities in the brain tissues of VD model rats. It has been reported 
that cerebrovascular levels vary with altered expression of fibrotic degrees (27). LDH levels are an indicator of oxidative stress, and a reduction in LDH levels is associated with alleviation of VD (28). Improvement of VD is also linked to reduced oxidative stress and apoptosis in the hippocampus of VD model rats, as well as decreased levels of Caspase-3 (29). Overall, the results of the present study suggested that miR-150 knockdown displayed therapeutic effects on VD model rats.

Subsequently, how miR-150 affected neurons in vitro was investigated. miR-150 antagomiR significantly downregulated Apaf1, p53 and Cyto C expression levels compared with antagomiR NC. p53 and Apaf1 are proapoptotic genes (30). Moreover, apoptosis can be further enhanced when p53 proteins are combined with Cyto $C$ (31). The aforementioned results supported the hypothesis that miR-150 knockdown suppresses cell apoptosis. Since ligustrazine is considered as a neuroprotective agent in the management of VD disease by suppressing PC12 cell apoptosis (32), it was also hypothesized that miR-150 knockdown might protect against VD by inhibiting PC12 cell apoptosis. A previous study demonstrated that miR-150 knockdown functions in the survival of cerebral cortical neurons (33). In the present study, compared with the empty vector group, HOXA1 overexpression significantly inhibited miR-150 mimic-induced PC12 cell apoptosis, which suggested that miR-150 might mediate the biological function of PC12 cells via HOXA1.

In the present study, further mechanistic investigations indicated that miR-150 targeted HOXA1. Gavalas et al (34) reported that HOXA1 or HOXB1 null mutants cause developmental disorders in the hindbrain. Subsequently, in 2007, Paraguison et al (35) demonstrated that polyhistidine variants of HOXA1 lead to decreased PBX homeobox 1 activity and inhibition of neuronal differentiation. In 2008, Martinez-Ceballos and Gudas (36) highlighted the necessity of HOXA1 in retinoic acid-induced embryonic stem cell differentiation to neurons. The aforementioned results indicated that HOXA1 is closely related to neuronal activity and development. A previous study reported that loss of HOXA1 could lead to a deficit in the neural crest (37). Of note, HOXA1 is regulated by different miRNAs in various diseases. For instance, miR-99a-5p regulates atherosclerotic progression via downregulation of HOXA1 mRNA and protein expression levels (15). Additionally, miR-10a-5p overexpression can suppress the HOXA1 expression in osteoarthritis (38). However, studies on the interaction between miR-150 and HOXA1 are limited.

The present study investigated the role of miR-150 in VD. Specifically, compared with the antagomiR NC group, miR-150 knockdown remarkably alleviated cognitive deficits and suppressed neuron apoptosis in the brain tissues of VD model rats. The results of the present study indicated that miR-150 knockdown might serve as a potential novel treatment strategy in VD-induced cognitive impairment. To the best of our knowledge, the present study identified an association between miR-150 and HOXA1 for the first time, and explored the effects of the miR-150/HOXA1 axis in VD in vitro and in vivo. Moreover, miR-150 might mediate other genes or signaling pathways in VD; therefore, the mechanisms underlying miRNA-modulated molecules in VD require further investigation. However, a limitation of this study includes the use of PC12 cells, which is a noradrenergic cell line derived from a rat pheochromocytoma, as the control. A more appropriate control cell line in future studies may further verify the authenticity of these results.

\section{Acknowledgements}

Not applicable.

\section{Funding}

The present study was supported by the Scientific Research Project of Heilongjiang Health Committee (grant nos. 2017-500 and 2017-496).

\section{Availability of data and materials}

The datasets used and/or analyzed during the current study are available from the corresponding author on reasonable request.

\section{Authors' contributions}

CW and XX contributed to designing the study and preparing the manuscript. HZ analyzed the data and edited the manuscript. XZ acquired and analyzed the data, and edited the manuscript. ZG conceptualized the study and reviewed the manuscript. CW, XX and HZ confirm the authenticity of all the raw data. All authors read and approved the final manuscript.

\section{Ethics approval and consent to participate}

The present study was approved by the Laboratory Animal Care and Use Committee of Heilongjiang Provincial Hospital (approval no. KY-2018-197) and performed according to the Guidelines for the Care and Use of Laboratory Animals published by the National Institutes of Health (17).

\section{Patient consent for publication}

Not applicable.

\section{Competing interests}

The authors declare that they have no competing interests.

\section{References}

1. O'Brien JT and Thomas A: Vascular dementia. Lancet 386: 1698-1706, 2015.

2. Jellinger KA and Attems J: Is there pure vascular dementia in old age? J Neurol Sci 299: 150-154, 2010.

3. Kalaria RN: The pathology and pathophysiology of vascular dementia. Neuropharmacology 134: 226-239, 2018.

4. Han F: Cerebral microvascular dysfunction and neurodegeneration in dementia. Stroke Vasc Neurol 4: 105-107, 2019.

5. Wolters FJ and Ikram MA: Epidemiology of vascular dementia. Arterioscler Thromb Vasc Biol 39: 1542-1549, 2019.

6. Farooq MU, Min J, Goshgarian C and Gorelick PB: Pharmacotherapy for vascular cognitive impairment. CNS Drugs 31: 759-776, 2017.

7. Vijayan M, Kumar S, Bhatti JS and Reddy PH: Molecular links and biomarkers of stroke, vascular dementia, and Alzheimer's disease. Prog Mol Biol Transl Sci 146: 95-126, 2017. 
8. Vijayan M and Reddy PH: Non-Coding RNAs based molecular links in type 2 diabetes, ischemic stroke, and vascular dementia. J Alzheimers Dis 75: 353-383, 2020.

9. Yuan M and Bi X: Therapeutic and diagnostic potential of microRNAs in vascular cognitive impairment. J Mol Neurosci 70 $1619-1628,2020$

10. Toyama K, Spin JM, Deng AC, Huang TT, Wei K, Wagenhäuser MU, Yoshino T, Nguyen H, Mulorz J, Kundu S, et al: MicroRNA-mediated therapy modulating blood-brain barrier disruption improves vascular cognitive impairment. Arterioscler Thromb Vasc Biol 38: 1392-1406, 2018.

11. Yu P, Venkat P, Chopp M, Zacharek A, Shen Y, Ning R, Liang L, Li W, Zhang L, Landschoot-Ward J, et al: Role of microRNA-126 in vascular cognitive impairment in mice. J Cereb Blood Flow Metab 39: 2497-2511, 2019.

12. Cohen A, Zinger A, Tiberti N, Grau GER and Combes V: Differential plasma microvesicle and brain profiles of microRNA in experimental cerebral malaria. Malar J 17: 192, 2018.

13. Ryan B, Williams JM and Curtis MA: Plasma MicroRNAs are altered early and consistently in a mouse model of tauopathy. Neuroscience 411: 164-176, 2019.

14. Jiang Q, Shan K, Qun-Wang X, Zhou RM, Yang H, Liu C, Li YJ, Yao J, Li XM, Shen Y, et al: Long non-coding RNA-MIAT promotes neurovascular remodeling in the eye and brain. Oncotarget 7: 49688-49698, 2016.

15. Han Z, Guan Y, Liu B, Lin Y, Yan Y, Wang H, Wang H and Jing B: MicroRNA-99a-5p alleviates atherosclerosis via regulating Homeobox A1. Life Sci 232: 116664, 2019.

16. Zhao J, He L and Yin L: lncRNA NEAT1 binds to MiR-339-5p to increase HOXA1 and alleviate ischemic brain damage in neonatal mice. Mol Ther Nucleic Acids 20: 117-127, 2020.

17. National Research Council (US) Institute for Laboratory Animal Research: Guide for the Care and Use of Laboratory Animals. National Academies Press, Washington, DC, 1996.

18. Wang XR, Shi GX, Yang JW, Yan CQ, Lin LT, Du SQ, Zhu W, He T, Zeng XH, Xu Q and Liu CZ: Acupuncture ameliorates cognitive impairment and hippocampus neuronal loss in experimental vascular dementia through Nrf2-mediated antioxidant response. Free Radic Biol Med 89: 1077-1084, 2015.

19. Xiao LY, Wang XR, Yang JW, Ye Y, Zhu W, Cao Y, Ma SM and Liu CZ: Acupuncture prevents the impairment of hippocampal LTP through $\beta 1-A R$ in vascular dementia rats. Mol Neurobiol 55: 7677-7690, 2018.

20. Livak KJ and Schmittgen TD: Analysis of relative gene expression data using real-time quantitative PCR and the 2(-Delta Delta C(T)) method. Methods 25: 402-408, 2001.

21. Zhang X, Cui SS, Wallace AE, Hannesson DK, Schmued LC, Saucier DM, Honer WG and Corcoran ME: Relations between brain pathology and temporal lobe epilepsy. J Neurosci 22 : 6052-6061, 2002.

22. Vannucci RC, Towfighi J and Vannucci SJ: Secondary energy failure after cerebral hypoxia-ischemia in the immature rat. J Cereb Blood Flow Metab 24: 1090-1097, 2004

23. Liu C, Guo Y, Zhao F, Qin H, Lu H, Fang L, Wang J and Min W: Potential mechanisms mediating the protective effects of a peptide from walnut (Juglans mandshurica Maxim.) against hydrogen peroxide induced neurotoxicity in PC12 cells. Food Funct 10: 3491-3501, 2019.

24. Xiao Y, Shen H, Li R, Zhou X, Xiao H and Yan J: A novel octapeptide derived from $G$ protein-coupled receptor 124 improves cognitive function via pro-angiogenesis in a rat model of chronic cerebral hypoperfusion-induced vascular dementia. Drug Des Devel Ther 13: 3669-3682, 2019.
25. Summers PM, Hartmann DA, Hui ES, Nie X, Deardorff RL, McKinnon ET, Helpern JA, Jensen JH and Shih AY: Functional deficits induced by cortical microinfarcts. J Cereb Blood Flow Metab 37: 3599-3614, 2017.

26. Shang Y, Cheng J, Qi J and Miao H: Scutellaria flavonoid reduced memory dysfunction and neuronal injury caused by permanent global ischemia in rats. Pharmacol Biochem Behav 82: 67-73, 2005.

27. Tong $\mathrm{XK}$ and Hamel E: Simvastatin restored vascular reactivity, endothelial function and reduced string vessel pathology in a mouse model of cerebrovascular disease. J Cereb Blood Flow Metab 35: 512-520, 2015.

28. Li X, Lu F, Li W, Qin L, Yao Y, Ge X, Yu Q, Liang X, Zhao D, Li $X$ and Zhang J: Edaravone injection reverses learning and memory deficits in a rat model of vascular dementia. Acta Biochim Biophys Sin (Shanghai) 49: 83-89, 2017.

29. Sun M, Shen X and Ma Y: Rehmannioside A attenuates cognitive deficits in rats with vascular dementia (VD) through suppressing oxidative stress, inflammation and apoptosis. Biomed Pharmacother 120: 109492, 2019.

30. Zhao F, Li H, Cao F, Chen X, Liang Y and Qiu L: Short-term developmental toxicity and potential mechanisms of the herbicide metamifop to zebrafish (Danio rerio) embryos. Chemosphere 236: 124590, 2019.

31. Chen X, Zhu Q, Xu X, Shen S, Zhang Y and Mo R: Sequentially site-specific delivery of apoptotic protein and tumor-suppressor gene for combination cancer therapy. Small 15: e1902998, 2019.

32. Zhao T, Fu Y, Sun H and Liu X: Ligustrazine suppresses neuron apoptosis via the Bax/Bcl-2 and caspase-3 pathway in PC12 cells and in rats with vascular dementia. IUBMB Life 70: 60-70, 2018

33. Lv H, Li J and Che YQ: MicroRNA-150 contributes to ischemic stroke through its effect on cerebral cortical neuron survival and function by inhibiting ERK1/2 axis via Mal. J Cell Physiol 234: $1477-1490,2019$

34. Gavalas A, Studer M, Lumsden A, Rijli FM, Krumlauf R and Chambon P: Hoxal and Hoxb1 synergize in patterning the hindbrain, cranial nerves and second pharyngeal arch. Development 125: 1123-1136, 1998.

35. Paraguison RC, Higaki $\mathrm{K}$, Yamamoto $\mathrm{K}$, Matsumoto $\mathrm{H}$, Sasaki T, Kato $\mathrm{N}$ and Nanba E: Enhanced autophagic cell death in expanded polyhistidine variants of HOXA1 reduces PBX1-coupled transcriptional activity and inhibits neuronal differentiation. J Neurosci Res 85: 479-487, 2007.

36. Martinez-Ceballos E and Gudas LJ: Hoxal is required for the retinoic acid-induced differentiation of embryonic stem cells into neurons. J Neurosci Res 86: 2809-2819, 2008.

37. Gouti M, Briscoe J and Gavalas A: Anterior Hox genes interact with components of the neural crest specification network to induce neural crest fates. Stem Cells 29: 858-870, 2011.

38. Ma Y, Wu Y, Chen J, Huang K, Ji B, Chen Z, Wang Q, Ma J, Shen S and Zhang J: miR-10a-5p promotes chondrocyte apoptosis in osteoarthritis by targeting HOXA1. Mol Ther Nucleic Acids 14: 398-409, 2019.

This work is licensed under a Creative Commons Attribution-NonCommercial-NoDerivatives 4.0 International (CC BY-NC-ND 4.0) License. 